\title{
Transient Electromagnetic Field Coupling to Buried Thin Wire Configurations: Antenna Model versus Transmission Line Approach in the Time Domain
}

\author{
Dragan Poljak, ${ }^{1}$ Silvestar Šesnić, ${ }^{1}$ Khalil El-Khamlichi Drissi, ${ }^{2}$ \\ Kamal Kerroum, ${ }^{2}$ and Sergey Tkachenko ${ }^{3}$ \\ ${ }^{1}$ University of Split, Split, Croatia \\ ${ }^{2}$ Université Blaise Pascal, Institut Pascal, Clermont-Ferrand, France \\ ${ }^{3}$ Department of Electrical Engineering and Information Technology, Otto-von-Guericke University Magdeburg, Magdeburg, Germany \\ Correspondence should be addressed to Dragan Poljak; dpoljak@fesb.hr
}

Received 2 November 2015; Revised 23 December 2015; Accepted 12 January 2016

Academic Editor: Luciano Tarricone

Copyright (C) 2016 Dragan Poljak et al. This is an open access article distributed under the Creative Commons Attribution License, which permits unrestricted use, distribution, and reproduction in any medium, provided the original work is properly cited.

The paper examines the antenna model for the transient analysis of electromagnetic field coupling to straight wire configurations buried in a lossy half-space. The wire antenna theory (AT) model is implemented directly in the time domain and it is based on the corresponding space-time Pocklington integrodifferential equation. The solution of the Pocklington equation is carried out analytically. The obtained results are compared against the results calculated via the transmission line (TL) approach. The TL approach is based on the telegrapher's equations, which are solved using the modified transmission line method (MTLM) and Finite Difference Time Domain (FDTD) technique, respectively. Some illustrative computational examples for buried straight wire scatterer and horizontal grounding electrode are given throughout this work.

\section{Introduction}

The analysis of transient electromagnetic field coupling to buried wire configurations is of interest in antennas, propagation, and electromagnetic compatibility (EMC) applications, for example, communications and power cables, geophysical investigations, grounding systems, and ground penetrating radars (GPR). This rather simple geometry itself could be used as a canonical reference structure to test numerical methods and codes, respectively. Furthermore, similar subject has been widely discussed in relevant literature regarding several EMC topics pertaining to interconnected lines, communications, and antennas; for example, see [1$3]$. The problem can be posed in either frequency or time domain by using the transmission line (TL) model or wire antenna theory (AT), also called the full-wave model [4, 5] with the latter approach being considered as a more rigorous one. In particular, the TL approach is considered to be quite sufficient approximation for long conductors with electrically small cross sections. However, TL model is not precise for finite length wires and high frequency excitations.

On the other hand, a principal drawback of the wire antenna model is rather high computational cost. Using certain enhanced TL models, one may overcome some limitations of the standard TL model restrictions [6].

The comparison between time domain wire antenna model and TL model pertaining to a single buried conductor has been presented in [7]. Time domain formulation used in $[8,9]$ arises from the wire antenna theory and is based on the corresponding time domain Pocklington integrodifferential equations for thin wires buried in a lossy half-space. The TL formulation is based on the telegrapher's equations. The Pocklington equation is solved analytically [9], while the telegrapher's equations are treated using the modified transmission line method (MTLM) [7].

Furthermore, transient analysis of a horizontal grounding electrode using both antenna theory and TL approximation 
has been reported in [10]. The Pocklington equation is solved analytically [11], while the TL equations are treated using the Finite Difference Time Domain (FDTD) method [10].

This paper revisits and fully extends the full-wave (AT) analysis methods of transient electromagnetic coupling to straight buried wire scatterer and horizontal grounding electrode configurations directly in the time domain presented in [6-11]. A comparison of the AT results with TL model results is given throughout the paper. Total leakage current has been determined directly in the time domain, as well. Note that frequency domain Pocklington equation is solved numerically via Galerkin-Bubnov Indirect Boundary Element Method [5], while the time domain Pocklington equation is solved analytically. Modelling of straight buried scatterer and horizontal grounding electrode, respectively, is outlined in different sections.

\section{Buried Wire Scatterers}

This section deals with a direct time domain analysis of a horizontal straight thin wire scatterer buried in a lossy halfspace and excited by a transmitted electromagnetic wave using the rigorous approach based on the antenna theory. More details could be found elsewhere, for example, in [9]. The AT approach is based on the space-time variant of the Pocklington integrodifferential approach. Though the Green function of a stratified medium is well-known and documented in a number of papers, for example, in [1214], the effect of the earth-air interface is taken into account via the simplified reflection coefficient arising from the Modified Image Theory (MIT) $[8,15,16]$. Namely, simple frequency domain formulas derived in [15] were transferred in the time domain in [16] and subsequently applied to the finite length geometry in [8]. The space-time Pocklington equation is solved analytically and the space-time current distribution is obtained. Once the current is determined, all other parameters of interest could be calculated, such as charge distribution, scattered field, and voltage, respectively. Such an analysis, related to the scattered voltages and charges, as well to the near field distribution due to uniform plane wave excitation, aiming to identify the scattering mechanisms of thin wires has been presented and has been also reported in [17]. The obtained numerical results are compared to the results calculated by means of the TL approach based on the corresponding set of telegrapher's equations handled via the MTLM. Moreover, the results obtained via time domain integral equation method are compared to the results obtained by numerically solving the space-frequency Pocklington equation via the Galerkin-Bubnov scheme of the Indirect Boundary Element Method (GB-IBEM) plus the inverse fast Fourier transform (IFFT) to obtain the corresponding transient response.

2.1. Antenna Theory Approach. The geometry of interest, related to the horizontal thin wire of length $L$ and radius a buried in a lossy ground at depth $d$, is shown in Figure 1.

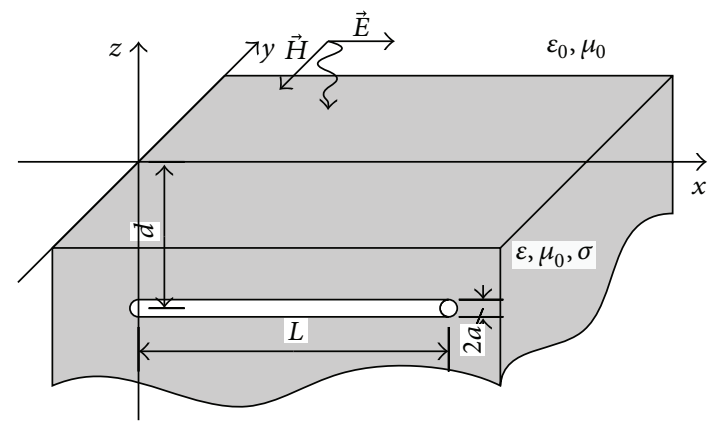

FIgURE 1: A horizontal thin wire buried in a lossy medium.

Direct time domain formulation for the transient analysis of horizontal straight buried wire is based on the space-time Pocklington integrodifferential equation given by [8]

$$
\begin{gathered}
\left(\mu \varepsilon \frac{\partial}{\partial t}+\mu \sigma\right) E_{x}^{\mathrm{tr}}(t)=-\left(\frac{\partial^{2}}{\partial x^{2}}-\mu \sigma \frac{\partial}{\partial t}-\mu \varepsilon \frac{\partial^{2}}{\partial t^{2}}\right) \\
\cdot\left[\frac{\mu}{4 \pi} \int_{0}^{L} I\left(x^{\prime}, t-\frac{R}{v}\right) \frac{e^{-\left(1 / \tau_{g}\right)(R / v)}}{R} d x^{\prime}-\frac{\mu}{4 \pi}\right. \\
\cdot \int_{0}^{t} \int_{0}^{L} \Gamma_{\mathrm{ref}}^{\mathrm{MIT}}(\tau) I\left(x^{\prime}, t-\frac{R^{*}}{v}-\tau\right) \\
\left.. \frac{e^{-\left(1 / \tau_{g}\right)\left(R^{*} / v\right)}}{R^{*}} d x^{\prime} d \tau\right],
\end{gathered}
$$

where $I\left(x^{\prime}, t-R / v\right)$ is the space-time dependent current to be determined, $E_{x}^{\mathrm{tr}}$ is the tangential transmitted field, and $\Gamma_{\text {ref }}^{\mathrm{MIT}}$ is the corresponding reflection coefficient arising from the Modified Image Theory $[8,15]$.

The distance from the source point in the wire axis to the observation point located on the wire surface is

$$
R=\sqrt{\left(x-x^{\prime}\right)^{2}+a^{2}}
$$

while the distance from the source point on the image wire to the observation point on the original wire according to the image theory is

$$
R^{*}=\sqrt{\left(x-x^{\prime}\right)^{2}+4 d^{2}} .
$$

Time constant $\tau_{g}$ and propagation velocity in the lossy medium $v$ are given by

$$
\begin{aligned}
\tau_{g} & =\frac{2 \varepsilon}{\sigma}, \\
v & =\frac{1}{\sqrt{\mu \varepsilon}},
\end{aligned}
$$

where $\sigma, \mu$, and $\varepsilon$ stand for conductivity, permittivity, and permeability, respectively.

The influence of the earth-air interface is taken into account via the reflection coefficient arising from the MIT given by $[8,15]$

$$
\Gamma_{\text {ref }}^{\mathrm{MIT}}(t)=-\left[\frac{\tau_{1}}{\tau_{2}} \delta(t)+\frac{1}{\tau_{2}}\left(1-\frac{\tau_{1}}{\tau_{2}}\right) e^{-t / \tau_{2}}\right],
$$


where $\delta(t)$ is the Dirac impulse and the corresponding time constants are

$$
\begin{gathered}
\tau_{1}=\frac{\varepsilon_{0}\left(\varepsilon_{r}-1\right)}{\sigma}, \\
\tau_{2}=\frac{\varepsilon_{0}\left(\varepsilon_{r}+1\right)}{\sigma} .
\end{gathered}
$$

Note that the reflection coefficient (6) represents rather simple characterization of the earth-air interface, taking into account only medium properties. Accuracy of (6) has been discussed elsewhere, for example, in $[8,9]$.

The analytical procedure documented in [6] for the case of delta pulse excitation yields the following expression:

$$
\begin{aligned}
& I(x, t)=\frac{4 \pi}{\mu}\left\{R\left(s_{\Psi}\right)\left[1-\frac{\cosh \left(\gamma_{\Psi}(L / 2-x)\right)}{\cosh \left(\gamma_{\Psi} L / 2\right)}\right]\right. \\
& \cdot e^{(t+a / v) s_{\Psi}}-\frac{\pi}{\mu \varepsilon L^{2}} \sum_{n=1}^{\infty} \frac{2 n-1}{ \pm \sqrt{b^{2}-4 c_{n}} s_{1,2 n} \Psi\left(s_{1,2 n}\right)} \\
& \left.\cdot \sin \frac{(2 n-1) \pi x}{L} e^{(t+a / v) s_{1,2 n}}\right\},
\end{aligned}
$$

where

$$
\begin{aligned}
& R\left(s_{\Psi}\right) \\
& =\frac{1}{2 \ln (L / 2 d)\left(s_{\Psi} /\left(s_{\Psi} \tau_{2}+1\right)\right)\left(\tau_{1}-\tau_{2}\left(\left(s_{\Psi} \tau_{1}+1\right) /\left(s_{\Psi} \tau_{2}+1\right)\right)\right)}, \\
& s_{\Psi}=-\frac{\ln (L / a)+\ln (L / 2 d)}{\tau_{1} \ln (L / a)+\tau_{2} \ln (L / 2 d)}, \\
& \Psi(s)=2\left(\ln \frac{L}{a}+\frac{s \tau_{1}+1}{s \tau_{1}+1} \ln \frac{L}{2 d}\right), \\
& \mathcal{Y}_{\Psi}=\sqrt{\mu \varepsilon\left(s_{\Psi}^{2}+b s_{\Psi}\right)}, \\
& s_{1,2 n}=\frac{1}{2}\left(-b \pm \sqrt{b^{2}-4 c_{n}}\right), \\
& b=\frac{\sigma}{\varepsilon}, \\
& c_{n}=\frac{(2 n-1)^{2} \pi^{2}}{\mu \varepsilon L^{2}}, \quad n=1,2,3, \ldots .
\end{aligned}
$$

Note that coefficients $R\left(s_{\Psi}\right)$ and $s_{\Psi}$ account for the properties of the medium, the dimensions of the wire, and the distance from the interface.

Expression (9) represents the impulse response of the wire scatterer. Consequently, the response to an arbitrary excitation requires convolution. In this paper, the normal incidence is considered; that is, the plane wave in the form of the double exponential function is assumed:

$$
E_{x}(t)=E_{0}\left(e^{-\alpha t}-e^{-\beta t}\right),
$$

where $E_{0}=1 \mathrm{~V} / \mathrm{m}, \alpha=4 * 10^{6} \mathrm{~s}^{-1}, \beta=4.78 * 10^{8} \mathrm{~s}^{-1}$.
The transmitted electric field exciting the buried wire in the Laplace domain is given by

$$
E_{x}^{\operatorname{tr}}(s)=\Gamma_{\operatorname{tr}}(s) E_{x}(s) e^{-\gamma d},
$$

where $\Gamma_{\text {tr }}(s)$ is the Fresnel transmission coefficient [9]

$$
\Gamma_{\text {tr }}(s)=\frac{2 \sqrt{s \varepsilon_{0}}}{\sqrt{s \mathcal{\varepsilon}+\sigma}+\sqrt{s \varepsilon_{0}}} .
$$

As the analytical convolution, that is, the time domain counterpart of (12), would be too complex to perform, the numerical convolution is carried out, as reported in [9].

2.2. Transmission Line Model. Frequency domain analysis of horizontal buried wire in a lossy medium excited via plane wave can be carried out by the transmissions line equations in the frequency domain [1]:

$$
\begin{aligned}
& \frac{\partial U(x, \omega)}{\partial x}+Z I(x, \omega)=E_{x}^{\mathrm{tr}}(x, \omega), \\
& \frac{\partial I(x, \omega)}{\partial x}+Y U(x, \omega)=0,
\end{aligned}
$$

where $U(x, \omega)$ and $I(x, \omega)$ are the induced voltage and current along the conductor, respectively, $Z(\omega)$ is the per-unit length impedance, and $Y(\omega)$ is the effective per-unit length admittance of the conductor, respectively.

The set of telegrapher's equations (14) is solved using the MTLM. The solution procedure is outlined in Appendix A.

Space-time voltage and current $u(x, t)$ and $i(x, t)$ are obtained as the inverse Fourier transform of space-frequency responses $U(x, \omega)$ and $I(x, \omega)$.

The per-unit lines parameters of buried horizontal wires can be calculated by using the approach proposed in [4]

\section{Results for Buried Wire Scatterer}

Some illustrative computational examples related to the transient response of a buried wire scatterer excited via transient plane wave (normal incidence) transmitted into the ground, as shown in Figure 1, are presented in Figures 2 and 3.

The results obtained using the AT approach in both frequency (FD) and time domain (TD), respectively, are compared to the results obtained by the MTLM approach. Figure 2 shows the transient current induced at the centre of the short wire ( $L=1 \mathrm{~m}, d=30 \mathrm{~cm}, a=5 \mathrm{~mm}$, and $\left.\varepsilon_{r}=10\right)$ for different values of ground conductivity.

Some discrepancies can be observed between the results obtained via AT-TD and TL methods versus AT-FD method, which is expected since two former methods have a similar approximation which is not valid for shorter wires.

Figure 3 shows the transient current induced at the centre of a longer wire $(L=10 \mathrm{~m})$. Again, there is a good agreement between the results obtained via different techniques.

Finally, Figure 4 shows the transient current at the centre of the same wire buried in a lossy ground with conductivity $\sigma=1 \mathrm{mS} / \mathrm{m}$ for different burial depths. 


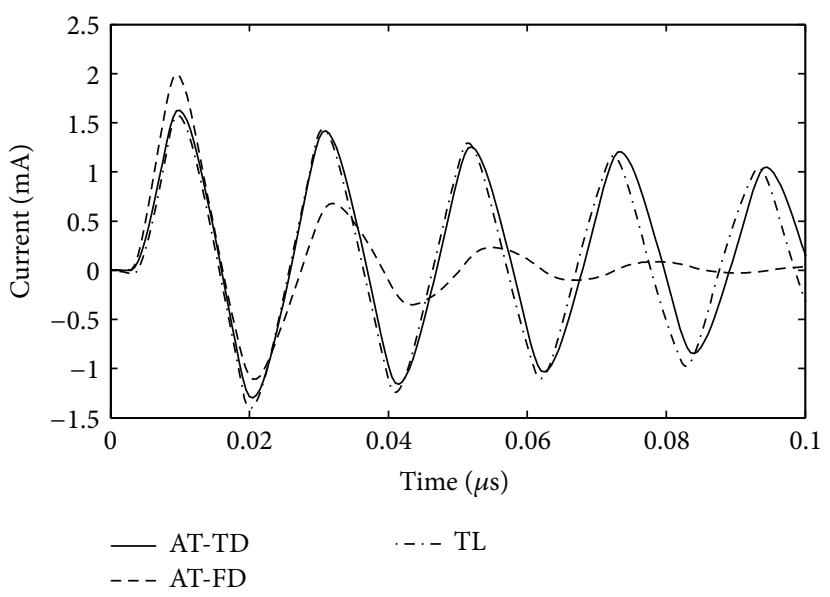

(a) $\sigma=1 \mathrm{mS} / \mathrm{m}$

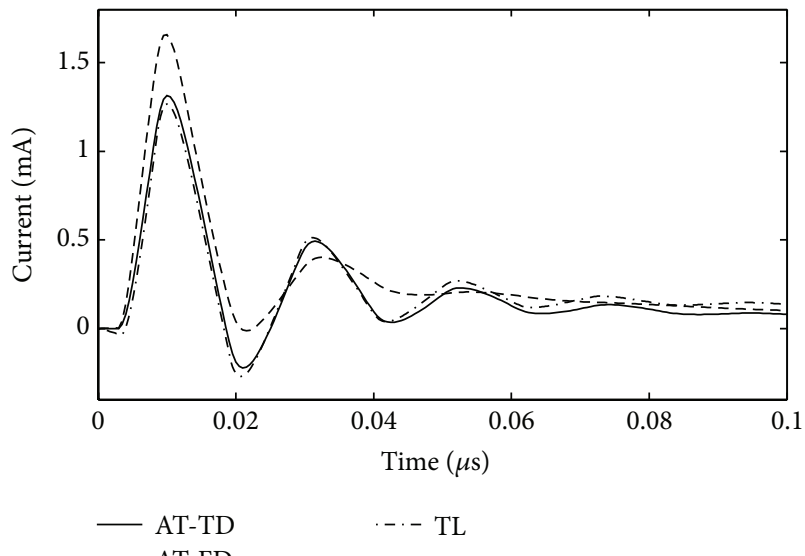

(b) $\sigma=10 \mathrm{mS} / \mathrm{m}$

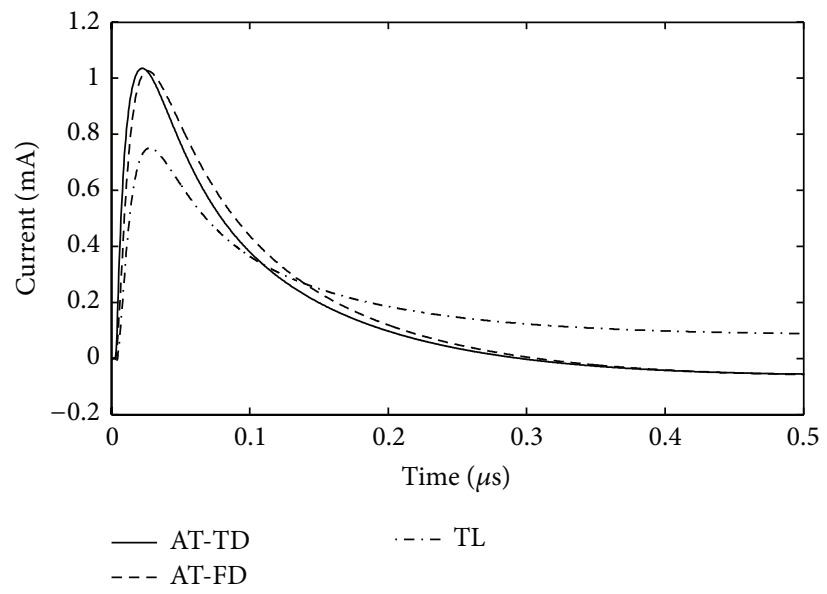

(c) $\sigma=100 \mathrm{mS} / \mathrm{m}$

Figure 2: Transient current at the centre of the straight wire: $L=1 \mathrm{~m}$ and $d=30 \mathrm{~cm}$.

The effect of the image wire is clearly dependent on depth $d$ and the medium conductivity $\sigma$. The greater the burial depth and soil conductivity are, the less significant the influence of the image wire in the air is.

The results calculated via different approaches agree satisfactorily in most cases. Some bigger differences exist only for the case of shorter wires and lower ground conductivity which was expected due to the nature of the transmission line model. AT-FD is considered to be the exact approach. The results obtained lead to the conclusion that the approximate solution of the time domain Pocklington equation directly in the time domain is close to the MTLM solution of the TD telegrapher's equations.

The mathematical details regarding the AT solution method could be found elsewhere, for example, in [5].

The computational cost is rather low if one uses analytical solution compared to the AT-FD and TL model, respectively. This is particularly of interest for more complex wire grid configurations.

\section{Horizontal Grounding Electrode}

A direct time domain analysis of a horizontal grounding electrode is of continuous interest in studies of lightning protection systems (LPS). The proposed geometry is of interest as a simple grounding system itself in some engineering applications and it could be also useful as a benchmark for testing other solution techniques.

This section deals with a transient analysis of a horizontal grounding wire immersed in a lossy half-space by means of a rigorous antenna theory and an approximate transmission line approach, respectively. The AT formulation is based on the homogeneous space-time Pocklington integrodifferential equation. The presence of the earth-air interface is, as previously shown, taken into account via the simplified reflection coefficient arising from the MIT. The TL approach is based on the corresponding set of space-time telegrapher's equations. The Pocklington equation is solved analytically while telegrapher's equations have been numerically solved via the Finite Difference Time Domain (FDTD) method. 


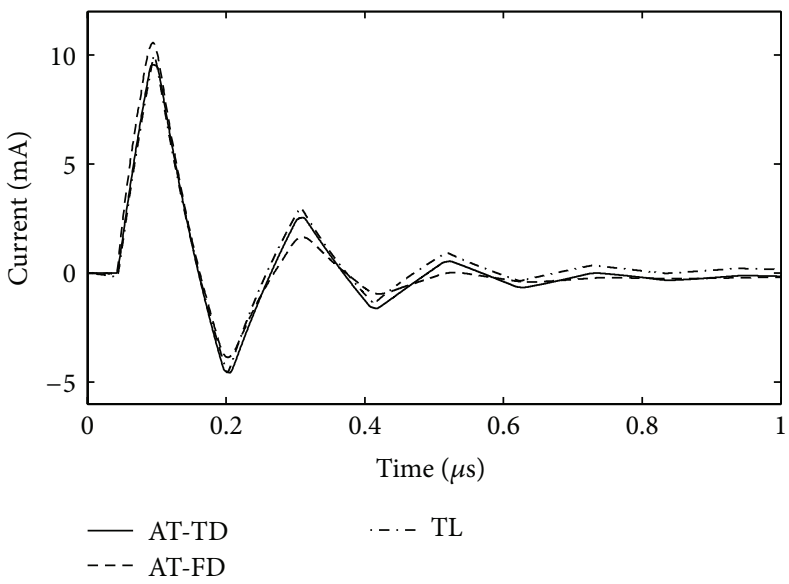

(a) $\sigma=1 \mathrm{mS} / \mathrm{m}$

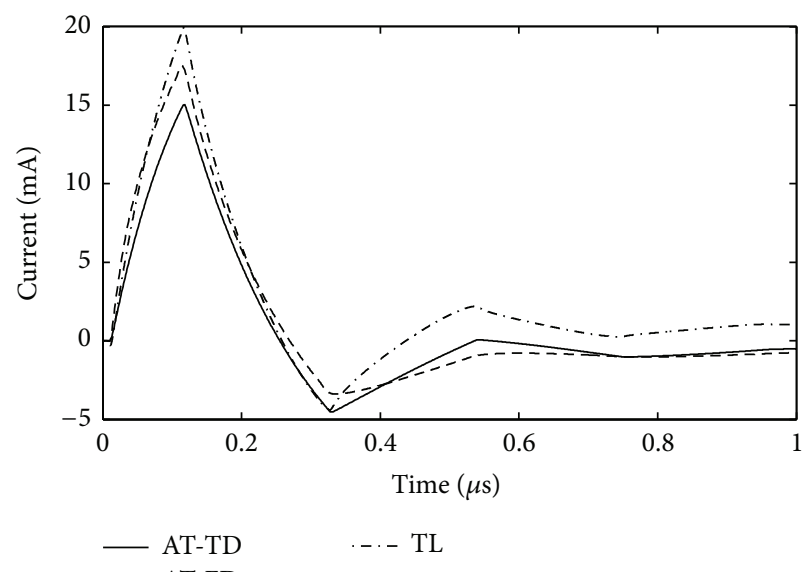

- - AT-FD

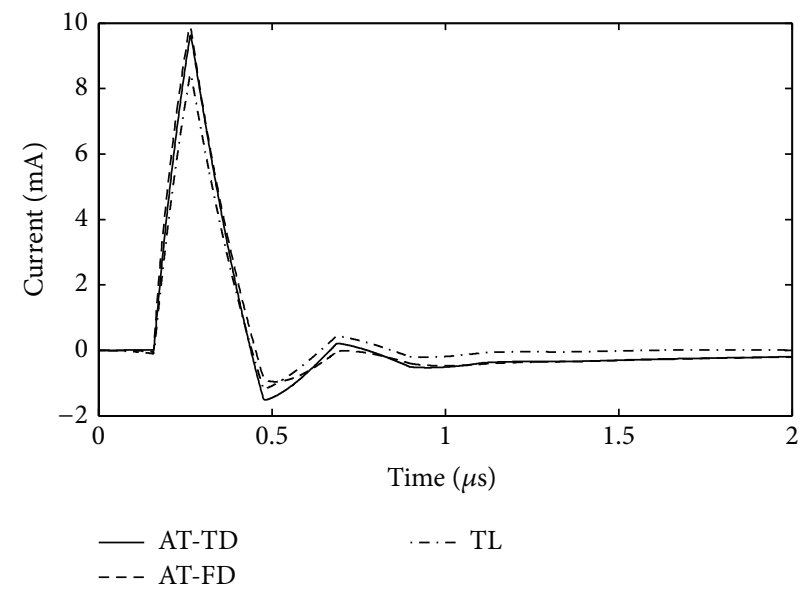

(c) $\sigma=100 \mathrm{mS} / \mathrm{m}$

FIgURE 3: Transient current at the centre of the straight wire: $L=10 \mathrm{~m}$ and $d=4 \mathrm{~m}$.

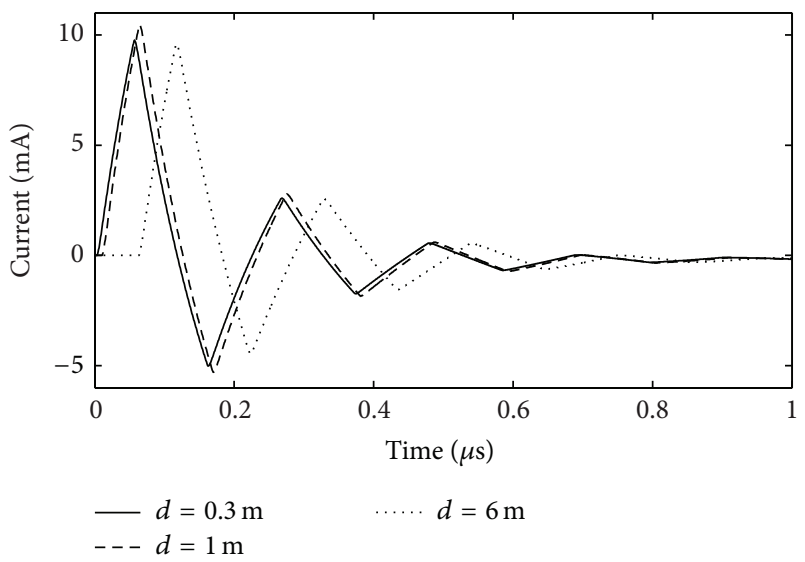

Figure 4: Transient current at the centre of the straight wire: $L=$ $10 \mathrm{~m}$ and $\sigma=1 \mathrm{mS} / \mathrm{m}$.

Finally, the knowledge of space-time current behaviour provides the calculation of the scattered voltage along the electrode $[18,19]$ and consequently determines the transient

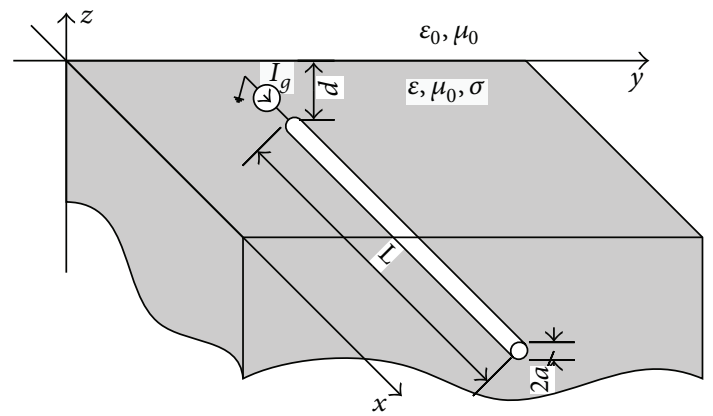

FIgURE 5: Horizontal grounding electrode buried in a lossy medium.

impedance, one of the most important parameters of grounding systems. This task will be addressed in a future work.

4.1. Antenna Theory Approach. The geometry of interest related to the horizontal grounding electrode of length $L$ and radius $a$, buried in a lossy medium of permittivity $\varepsilon$ and conductivity $\sigma$ at depth $d$, is shown in Figure 5. The electrode is energized at one end with an equivalent current source. 
Direct time domain analysis of the horizontal grounding electrode is based on the following homogeneous space-time Pocklington integrodifferential equation [11]:

$$
\begin{gathered}
\left(\frac{\partial^{2}}{\partial x^{2}}-\mu \sigma \frac{\partial}{\partial t}-\mu \varepsilon \frac{\partial^{2}}{\partial t^{2}}\right) \cdot\left[\frac{\mu}{4 \pi} \int_{0}^{L} I\left(x^{\prime}, t-\frac{R}{v}\right)\right. \\
\cdot \frac{e^{-\left(1 / \tau_{g}\right)(R / v)}}{R} d x^{\prime}-\frac{\mu}{4 \pi} \int_{0}^{t} \int_{0}^{L} \Gamma_{\mathrm{ref}}^{\mathrm{MIT}}(\tau) \\
\left.\cdot I\left(x^{\prime}, t-\frac{R^{*}}{v}-\tau\right) \frac{e^{-\left(1 / \tau_{g}\right)\left(R^{*} / v\right)}}{R^{*}} d x^{\prime} d \tau\right]=0,
\end{gathered}
$$

where $I\left(x^{\prime}, t-R / v\right)$ is the space-time current along the electrode and $\Gamma_{\text {ref }}^{\mathrm{MIT}}$ is the reflection coefficient (6) while $R$, $R^{*}, \tau_{g}, v, \tau_{1}$, and $\tau_{2}$ are given with expressions (2) to (8), respectively.

When dealing with the transient analysis of grounding electrodes, the excitation function is usually defined in terms of the equivalent current generator representing the lightning strike current.

One of the simplest representations of the lightning current, used in this paper, as well, is the double exponential pulse [20]:

$$
I_{g}(t)=I_{0}\left(e^{-\alpha t}-e^{-\beta t}\right)
$$

where $I_{0}=1.1043 \mathrm{~A}, \alpha=0.07924 * 10^{7} \mathrm{~s}^{-1}$, and $\beta=4.0011 *$ $10^{7} \mathrm{~s}^{-1}$.

The space-time current flowing along the horizontal grounding electrode due to the double exponential current source (16) is given by [1]

$$
\begin{aligned}
I(x, t)= & \frac{2 \pi I_{0}}{\mu \varepsilon L^{2}} \sum_{n=1}^{\infty} \frac{(-1)^{n-1} n}{b^{2}-4 c_{n}} \sin \frac{n \pi(L-x)}{L} \\
& \cdot\left(\frac{e^{s_{1,2 n} t}-e^{-\alpha t}}{s_{1,2 n}+\alpha}-\frac{e^{s_{1,2 n} t}-e^{-\beta t}}{s_{1,2 n}+\beta}\right),
\end{aligned}
$$

where

$$
\begin{aligned}
s_{1,2 n} & =\frac{1}{2}\left(-b \pm \sqrt{b^{2}-4 c_{n}}\right), \\
b & =\frac{\sigma}{\varepsilon} \\
c_{n} & =\frac{n^{2} \pi^{2}}{\mu \varepsilon L^{2}}, \quad n=1,2,3, \ldots .
\end{aligned}
$$

More mathematical details regarding the analytical solution of (15) are available in [11].

4.2. Transmission Line Model. Using the framework of the transmission line theory, the current and voltage induced along the horizontal grounding electrode can be obtained by solving the set of telegrapher's equations [21]:

$$
\begin{gathered}
\frac{\partial v(x, t)}{\partial x}+R i(x, t)+L \frac{\partial i(x, t)}{\partial t}=0, \\
\frac{\partial i(x, t)}{\partial x}+G v(x, t)+C \frac{\partial v(x, t)}{\partial t}=0,
\end{gathered}
$$

where $v(x, t)$ and $i(x, t)$ are induced voltage and current along the conductor, respectively, and $R$ is the per-unit length series resistance. $L, G$, and $C$ are the effective per-unit length inductance, conductance, and capacitance of the conductor, respectively. The zero-current conditions at the open wire ends are assumed; that is, $i(0, t)=0$ and $i(L, t)=0$.

Furthermore, the per-unit lines parameters of buried horizontal wires can be calculated using the following expressions [20]:

$$
\begin{aligned}
& L=\frac{\mu_{0}}{2 \pi} \ln \left(\frac{2 l}{\sqrt{2 a d}}-1\right), \\
& G=\frac{2 \pi \sigma}{\ln (2 l / \sqrt{2 a d}-1)}, \\
& C=\frac{2 \pi \varepsilon_{0} \varepsilon_{r}}{\ln (2 l / \sqrt{2 a d}-1)} .
\end{aligned}
$$

As in antenna theory, the electrode is assumed to be perfectly conducting (PEC); that is, $R=0$. Note that $l, a$, and $d$ are the length, radius, and depth, respectively, of the buried horizontal electrode, while $\mu_{0}, \varepsilon_{r}$, and $\sigma$ are the permeability of air, the relative permittivity, and the conductivity of the ground, respectively.

The solution of telegrapher's equations (19) is carried out using the Finite Difference Time Domain (FDTD) method [7] and it is outlined in Appendix B.

4.3. The Leakage Current. One of the parameters that can be useful in the analysis of the grounding electrode properties is the leakage current density [22].

The concept of leakage current density flowing radially from the electrode is depicted in Figure 6.

This current density can be evaluated as the product of the radial field component $E_{\rho}$

$$
E_{\rho}=-\frac{1}{j \omega \varepsilon_{\mathrm{eff}} \cdot 2 \pi \rho} \frac{\partial I(z)}{\partial z}
$$

and the soil conductivity $\sigma$.

Thus, the leakage current density is given by [12]

$$
J_{l}=\sigma \cdot E_{\rho}=-\frac{\sigma}{j \omega \varepsilon_{\mathrm{eff}} \cdot 2 \pi \rho} \frac{\partial I(z)}{\partial z} .
$$

The total current flowing out of the electrode can be obtained by integrating the current density along the cylinder

$$
\begin{aligned}
I_{l, \text { tot }} & =\int_{S} \vec{J}_{l} \cdot d \vec{S} \\
& =-\frac{\sigma}{j \omega \varepsilon_{\mathrm{eff}} \cdot 2 \pi} \int_{0}^{2 \pi} \int_{0}^{L} \frac{1}{\rho} \frac{\partial I(z)}{\partial z} \rho d \phi d z
\end{aligned}
$$




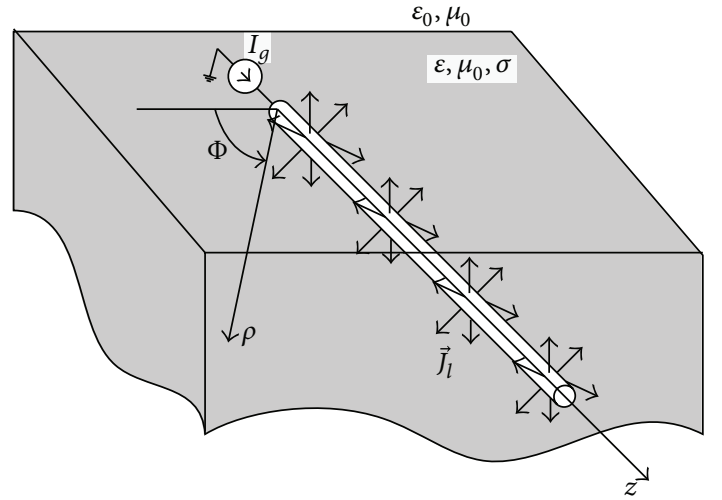

FIGURE 6: The leakage current density.

and is given by

$$
I_{l, \text { tot }}=\frac{\sigma}{j \omega \varepsilon_{\text {eff }}} I(0) .
$$

In the Laplace domain, (24) becomes

$$
I_{l, \text { tot }}(s)=\frac{1}{1+s T} I_{g}(s),
$$

where $T=\varepsilon / \sigma$.

It is convenient to rewrite (25), as follows:

$$
I_{l, \text { tot }}(s)=H(s) \cdot I_{g}(s),
$$

where $H(s)$ can be written in the form

$$
H(s)=\frac{1 / T}{s+1 / T} .
$$

The product from (26) in the Laplace domain corresponds to the convolution in the time domain:

$$
i_{l, \text { tot }}(t)=\int_{0}^{t} h(t-\tau) i_{g}(\tau) d \tau,
$$

where $i_{g}(t)$ is given by (16) and $h(t)$ is the time domain counterpart of (27):

$$
h(t)=\frac{1}{T} e^{-t / T} .
$$

Inserting (16) and (29) into (28) yields

$$
i_{l, \text { tot }}(t)=\int_{0}^{t} \frac{1}{T} e^{-(t-\tau) / T} I_{0}\left(e^{-\alpha \tau}-e^{-\beta \tau}\right) d \tau .
$$

The solution of (30) is straightforward and can be written in the form

$$
\begin{aligned}
& i_{l, \text { tot }}(t)=\frac{I_{0}}{T} e^{-t / T} \cdot\left\{\frac{1}{\alpha-1 / T}\left[1-e^{-t(\alpha-1 / T)}\right]\right. \\
& \left.-\frac{1}{\beta-1 / T}\left[1-e^{-t(\beta-1 / T)}\right]\right\} .
\end{aligned}
$$

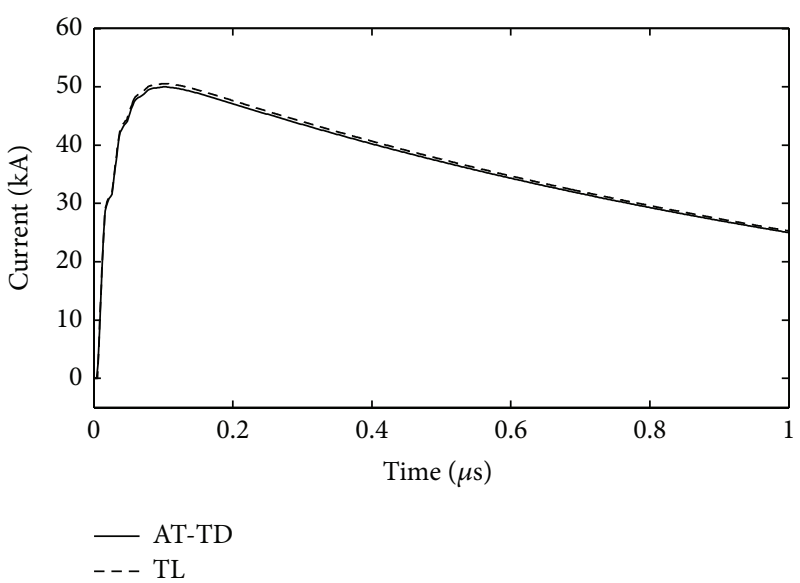

FIGURE 7: Transient current at the centre of the electrode: $L=1 \mathrm{~m}$, $\sigma=10 \mathrm{mS} / \mathrm{m}$, and $0.1 / 1 \mu$ s pulse.

\section{Results for the Horizontal Grounding Electrode}

All examples are related to the horizontal electrode of radius $a=5 \mathrm{~mm}$, buried in a lossy half-space with relative permittivity $\varepsilon_{r}=10$ at depth $d=0.5 \mathrm{~m}$. The grounding electrode is energized with the double exponential current source with $I_{0}=100 \mathrm{kA}$. The transient current is determined by (17). The obtained analytical results are compared to the numerical results obtained by solving telegrapher's equations (19) via FDTD method. Figure 7 shows the transient response at the centre of the $1 \mathrm{~m}$ long electrode buried in a lossy medium $(\sigma=10 \mathrm{mS} / \mathrm{m})$ and excited by the $0.1 / 1 \mu$ s pulse defined by the following set of parameters:

$$
\begin{aligned}
& \alpha=0.07924 \cdot 10^{7}(1 / \mathrm{s}), \\
& \beta=4.0011 \cdot 10^{7}(1 / \mathrm{s}) .
\end{aligned}
$$

Figures 8 and 9 show the transient response at the centre of the $10 \mathrm{~m}$ long electrode for the soil conductivity $\sigma=1 \mathrm{mS} / \mathrm{m}$ and $\sigma=10 \mathrm{mS} / \mathrm{m}$, respectively.

The transient response at the centre of the $20 \mathrm{~m}$ long electrode buried in the lossy medium with the conductivity $\sigma=1 \mathrm{mS} / \mathrm{m}$ is shown in Figure 10 .

It can be observed that the results obtained by means of the AT and TL approach, respectively, agree rather satisfactorily.

Therefore, it can be concluded that the approximations adopted within the analytical solution procedure of integrodifferential equation (15) to a certain extent correspond to the approximations adopted in the TL model itself.

This is an important conclusion as the proposed analytical formula could be used instead of FDTD solution of telegrapher's equations in the time domain. Moreover, the space-time expression provides a further calculation of the transient voltage along the electrode and consequently transient impedance without using FFT which could be a cause of significant errors within the frequency domain approach. This task will be addressed in the future work. 


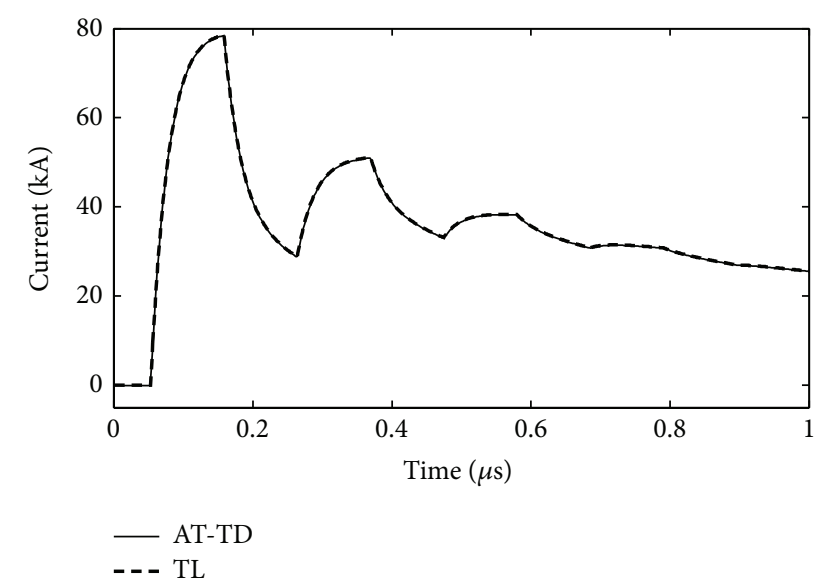

Figure 8: Transient current at the centre of the electrode: $L=10 \mathrm{~m}$, $\sigma=1 \mathrm{mS} / \mathrm{m}$, and $0.1 / 1 \mu$ s pulse.

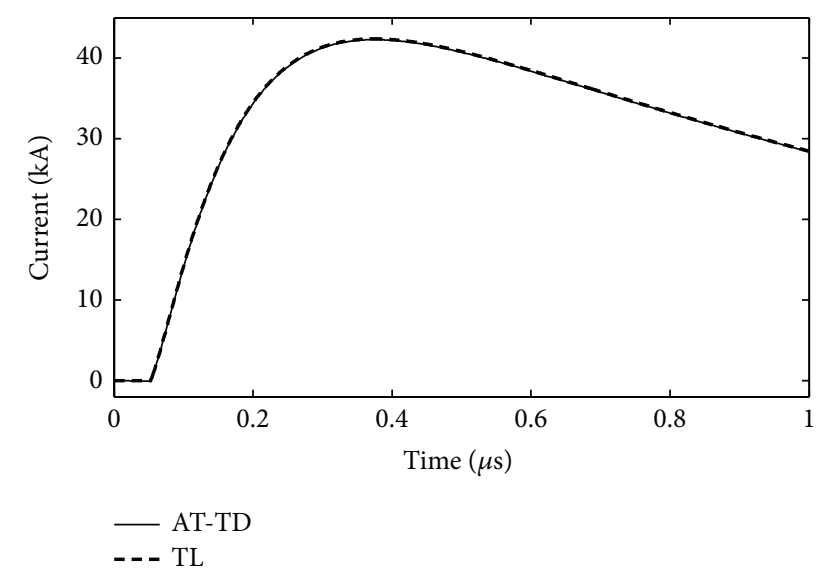

FIGURE 9: Transient current at the centre of the electrode: $L=10 \mathrm{~m}$, $\sigma=10 \mathrm{mS} / \mathrm{m}$, and $0.1 / 1 \mu$ s pulse.

Regarding electrode length, the analytical solution of the current waveform seems to improve for higher electrode length $(L=10 \mathrm{~m})$. Such behaviour is expected, as the approximations in analytical solution of the thin wire integral equation are rather close to certain assumption adopted within the transmission line (TL) approach [11].

Figure 11 shows a time dependent total leakage current flowing out of the electrode in the normal direction, for the ground permittivity $\varepsilon_{r}=10$ and different values of ground conductivity. The double exponential excitation (16) is related to the $0.1 / 1 / \mu \mathrm{s}, 1 \mathrm{~A}$ pulse.

It is visible from Figure 11 that the higher the conductivity the faster the die-off of the total leakage current curve. It can also be observed from Figure 11 that the leakage current is around 5 orders of magnitude lower than the axial current along the electrode. This is a direct consequence of the assumption that the wire is perfectly conducting.

\section{Conclusion}

Direct time domain analysis of the transient behaviour of the buried wire scatterer and horizontal grounding electrode

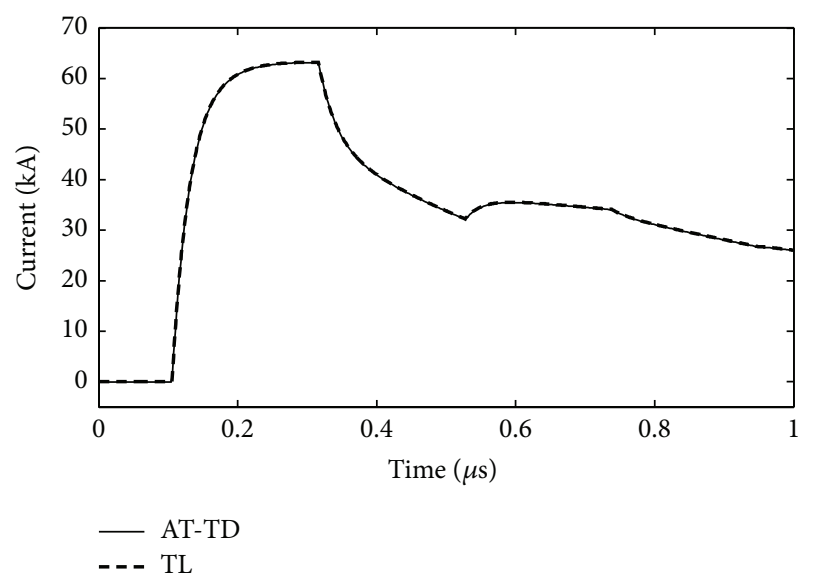

FIGURE 10: Transient current at the centre of the electrode: $L=20 \mathrm{~m}$, $\sigma=1 \mathrm{mS} / \mathrm{m}$, and $0.1 / 1 \mu$ s pulse.

based on the rigorous antenna theory model has been discussed in this work. The AT approach is based on the corresponding space-time Pocklington integrodifferential equation. The influence of the earth-air interface is taken into account via the simplified reflection coefficient arising from the Modified Image Theory. The corresponding Pocklington equation is solved analytically. The obtained results are compared to the results obtained via the approximate TL approach, based on the set of the corresponding telegrapher's equations solved via Modified Transmission Line Method and the Finite Difference Time Domain method, respectively. In the case of wire scatterer, AT-TD results are also compared to the results obtained via the AT-FD approach combined with IFFT. The results calculated via different approaches agree rather satisfactorily. A trade-off between the AT-TD analytical approach, AT-FD numerical approach, and numerical solution of the telegrapher's equation has been carried out throughout the paper.

\section{Appendices}

\section{A. MTLM Solution of Telegrapher's Equations}

The set of telegrapher's equations [1] is as follows:

$$
\begin{aligned}
\frac{\partial v(x, t)}{\partial x}+z(t) * i(x, t) & =E_{x}^{\mathrm{tr}}(x, t), \\
\frac{\partial i(x, t)}{\partial x}+y(t) * v(x, t)+C \frac{\partial v(x, t)}{\partial t} & =0
\end{aligned}
$$

where $*$ is the convolution product. In the frequency domain (A.1) can be written as follows [1]:

$$
\begin{aligned}
& \frac{\partial U(x, \omega)}{\partial x}+Z I(x, \omega)=E_{x}^{\mathrm{tr}}(x, \omega), \\
& \frac{\partial I(x, \omega)}{\partial x}+Y U(x, \omega)=0 .
\end{aligned}
$$




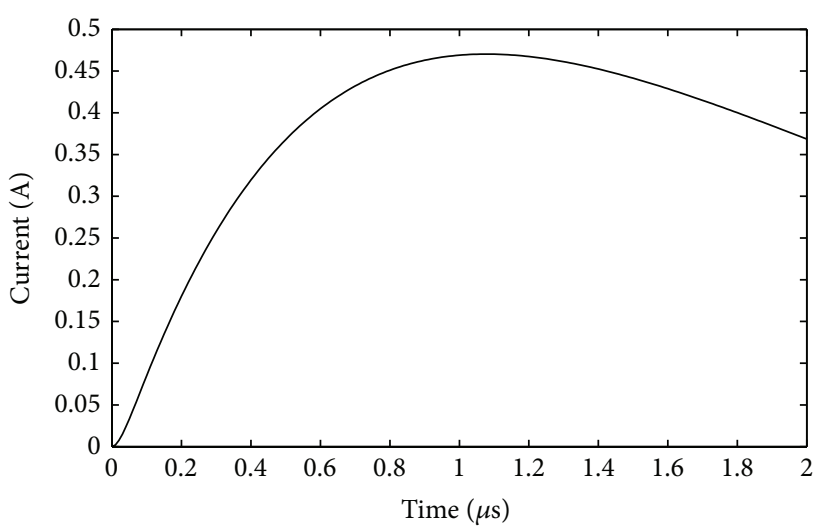

(a) $\sigma=0.1 \mathrm{mS} / \mathrm{m}$

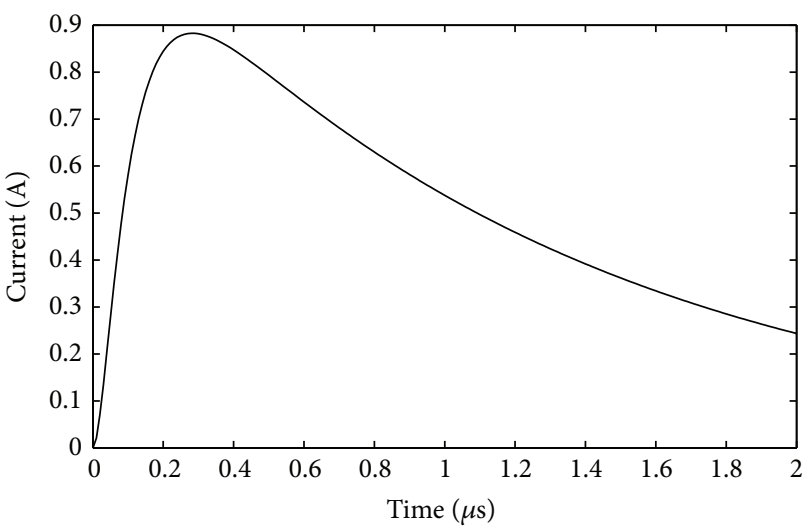

(b) $\sigma=1 \mathrm{mS} / \mathrm{m}$

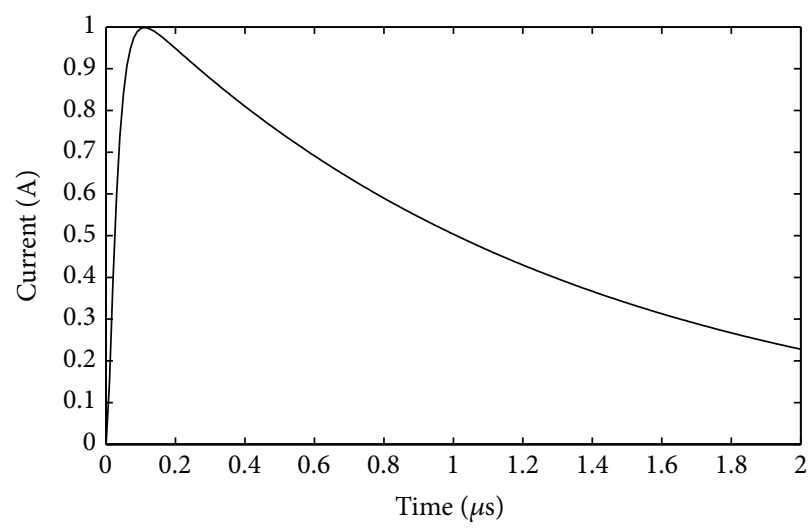

(c) $\sigma=10 \mathrm{mS} / \mathrm{m}$

FIGURE 11: Total leakage current versus time.

The solution of transmission line equations for a buried wire excited by an external field $E_{x}^{\mathrm{tr}}(\omega)$ transmitted into the ground, given by (12), can be written in the form

$$
I(x, \omega)=A(\omega) e^{-\Gamma x}+B(\omega) e^{\Gamma x}+\frac{E_{x}^{\mathrm{tr}}(\omega)}{Z(\omega)},
$$

where $A(\omega)$ and $B(\omega)$ are determined from the zero-current conditions at the wire ends

$$
I(0, \omega)=I(L, \omega)=0
$$

and are given by

$$
\begin{aligned}
& A(\omega)=\frac{E_{x}^{\mathrm{tr}}(\omega)}{Z(\omega)} \frac{\left(1-e^{\Gamma L}\right)}{\left(e^{\Gamma L}-e^{-\Gamma L}\right)}, \\
& B(\omega)=-\frac{E_{x}^{\mathrm{tr}}(\omega)}{Z(\omega)} \frac{\left(1-e^{-\Gamma L}\right)}{\left(e^{\Gamma L}-e^{-\Gamma L}\right)} .
\end{aligned}
$$

Per-unit length parameters $Z(\omega)$ and $Y(\omega)$ are defined by relation

$$
Z[\Gamma(\omega)] \cdot Y[\Gamma(\omega)]=[\Gamma(\omega)]^{2},
$$

where [7]

$$
\begin{aligned}
& Z(\Gamma) \\
& \quad=\frac{j \omega \mu_{0}}{2 \pi}\left[K_{0}\left(\gamma_{1} a\right)-K_{0}\left(\gamma_{1}(2 d-a)\right)+I_{1}(\Gamma)\right], \\
& Y(\Gamma)=\frac{j 2 \pi \omega \varepsilon_{\text {eff }}}{K_{0}\left(\gamma_{1} a\right)-K_{0}\left(\gamma_{1}(2 d-a)\right)+k_{1}^{2} I_{2}(\Gamma)} .
\end{aligned}
$$

Furthermore, $I_{1}$ and $I_{2}$ are determined by integrals

$$
\begin{aligned}
& I_{1}(\Gamma)=\int_{-\infty}^{+\infty} \frac{e^{-2 u_{1} d}}{u_{1}+u_{2}} d \lambda . \\
& I_{2}(\Gamma)=\int_{-\infty}^{+\infty} \frac{e^{-2 u_{1} d}}{k_{2}^{2} u_{1}+k_{1}^{2} u_{2}} d \lambda,
\end{aligned}
$$

where $K_{0}$ is zero order Bessel function of the second kind, while $u_{1}$ and $u_{2}$ are given by

$$
\begin{aligned}
& u_{1}=\left(\lambda^{2}-\Gamma^{2}-k_{1}^{2}\right)^{1 / 2}=\left(\lambda^{2}+\gamma_{1}^{2}\right)^{1 / 2}, \\
& u_{2}=\left(\lambda^{2}-\Gamma^{2}-k_{2}^{2}\right)^{1 / 2}=\left(\lambda^{2}+\gamma_{2}^{2}\right)^{1 / 2},
\end{aligned}
$$


where $k_{1}$ and $k_{2}$ are the propagation constants of a lossy ground and air, respectively, and are expressed as follows:

$$
\begin{aligned}
& k_{1}^{2}=\omega^{2} \mu_{0} \varepsilon_{\mathrm{eff}}, \\
& k_{2}^{2}=\omega^{2} \mu_{0} \varepsilon_{0},
\end{aligned}
$$

with

$$
\varepsilon_{\mathrm{eff}}=\varepsilon_{0} \varepsilon_{r}-j \frac{\sigma}{\omega} .
$$

Space-time current $I(x, t)$ is obtained as the inverse Fourier transform of space-frequency response $I(x, \omega)$.

\section{B. FDTD Solution of Telegrapher's Equations}

A set of telegrapher's equations

$$
\begin{gathered}
\frac{\partial v(x, t)}{\partial x}+R i(x, t)+L \frac{\partial i(x, t)}{\partial t}=0, \\
\frac{\partial i(x, t)}{\partial x}+G v(x, t)+C \frac{\partial v(x, t)}{\partial t}=0
\end{gathered}
$$

are solved using the FDTD method.

Discretizing the electrode into $N_{s}$ segments, with segment length $\Delta x$ and the time interval of interest into $n t$ sections, with increment $\Delta t$, it follows that

$$
\begin{aligned}
& v_{k}^{n+1}=D\left[E \cdot v_{k}^{n}-\left(i_{k}^{n+1 / 2}-i_{k-1}^{n+1 / 2}\right)\right], \\
& \text { for } k=2,3, \ldots, N, \\
& i_{k}^{n+3 / 2}=A\left[B \cdot i_{k}^{n+1 / 2}-\left(v_{k+1}^{n+1}-v_{k}^{n+1}\right)\right], \\
& \text { for } k=1,2, \ldots, N,
\end{aligned}
$$

where

$$
\begin{aligned}
v_{k}^{n} & =v[(k-1) \Delta x, n \Delta t], \text { for } n=0,1, \ldots, n t, \\
i_{k}^{n} & =i\left[\left(k-\frac{1}{2}\right) \Delta x, n \Delta t\right], \text { for } n=0,1, \ldots, n, \\
A & =\left(\frac{\Delta x}{\Delta t} l+\Delta x \frac{r}{2}\right)^{-1}, \\
B & =\left(\frac{\Delta x}{\Delta t} l-\Delta x \frac{r}{2}\right)^{-1}, \\
D & =\left(\frac{\Delta x}{\Delta t} c+\Delta x \frac{g}{2}\right)^{-1}, \\
E & =\left(\frac{\Delta x}{\Delta t} c-\Delta x \frac{g}{2}\right)^{-1} .
\end{aligned}
$$

Incorporating the appropriate boundary conditions for voltages at the wire ends, $v_{1}^{n}, v_{n x+1}^{n}$ are evaluated from the following expressions:

$$
\begin{aligned}
v_{1}^{n+1} & =D\left[E \cdot v_{1}^{n}-2 i_{1}^{n+1 / 2}+\left(i_{g}^{n+1}-i_{g}^{n}\right)\right], \\
v_{n x+1}^{n+1} & =D\left(E \cdot v_{n x}^{n}+2 i_{n x}^{n+1 / 2}\right) .
\end{aligned}
$$

The current excitation $I_{g}^{n}$ at the time instant $t=n \Delta t$ is given by (16).

\section{Conflict of Interests}

The authors declare that there is no conflict of interests regarding the publication of this paper.

\section{References}

[1] G. S. Shinh, N. M. Nakhla, R. Achar, M. S. Nakhla, A. Dounavis, and I. Erdin, "Fast transient analysis of incident field coupling to multiconductor transmission lines," IEEE Transactions on Electromagnetic Compatibility, vol. 48, no. 1, pp. 57-73, 2006.

[2] P. Bernardi, R. Cicchetti, and C. Pirone, "Transient response of a microstrip line circuit excited by an external electromagnetic source," IEEE Transactions on Electromagnetic Compatibility, vol. 34, no. 2, pp. 100-108, 1992.

[3] M. Leone and H. L. Singer, "On the coupling of an external electromagnetic field to a printed circuit board trace," IEEE Transactions on Electromagnetic Compatibility, vol. 41, no. 4, pp. 418-424, 1999.

[4] F. M. Tesche, M. Ianoz, and T. Karlsson, EMC Analysis Methods and Computational Models, John Wiley \& Sons, New York, NY, USA, 1997.

[5] D. Poljak, Advanced Modeling in Computational Electromagnetic Compatibility, Wiley-Interscience, Hoboken, NJ, USA, 2007.

[6] D. Poljak, V. Doric, S. Antonijevic, K. El Khamlichi Drissi, and K. Kerroum, "Electromagnetic field coupling to overhead wires: comparison of wire antenna and transmission line model in the frequency and time domain," in Proceedings of the 19th International Conference on Applied Electromagnetics and Communications (ICECom '07), pp. 1-4, IEEE, Dubrovnik, Croatia, September 2007.

[7] D. Poljak, S. Sesnic, K. El Khamlichi Drissi, and K. Kerroum, "Transient response of a buried wire," in Proceedings of the IEEE 21st International Conference on Applied Electromagnetics and Communications (ICECom '13), pp. 1-4, Dubrovnik, Croatia, October 2013.

[8] D. Poljak and N. Kovac, "Time domain modeling of a thin wire in a two-media configuration featuring a simplified reflection/transmission coefficient approach," Engineering Analysis with Boundary Elements, vol. 33, no. 3, pp. 283-293, 2009.

[9] S. Šesnić, D. Poljak, and S. Tkachenko, "Time domain analytical modeling of a straight thin wire buried in a lossy medium," Progress in Electromagnetics Research, vol. 121, pp. 485-504, 2011.

[10] D. Poljak, S. Sesnic, S. V. Tkachenko, K. El Khamlichi Drissi, and K. Kerroum, "Time domain analysis of the horizontal grounding electrode: antenna theory approach versus transmission line approximation," in Proceedings of the International Symposium on Electromagnetic Compatibility (EMC Europe '14), pp. 181-185, IEEE, Gothenburg, Sweden, September 2014.

[11] S. Šesnić, D. Poljak, and S. V. Tkachenko, "Analytical modeling of a transient current flowing along the horizontal grounding electrode," IEEE Transactions on Electromagnetic Compatibility, vol. 55, no. 6, pp. 1132-1139, 2013.

[12] K. A. Michalski and J. R. Mosig, "Multilayered media green's functions in integral equation formulations," IEEE Transactions on Antennas and Propagation, vol. 45, no. 3, pp. 508-519, 1997.

[13] P. Ylä-Oijala and M. Taskinen, "Efficient formulation of closedform Green's functions for general electric and magnetic sources in multilayered media," IEEE Transactions on Antennas and Propagation, vol. 51, no. 8, pp. 2106-2115, 2003.

[14] R. R. Boix, A. L. Fructos, and F. Mesa, "Closed-form uniform asymptotic expansions of Green's functions in layered media," 
IEEE Transactions on Antennas and Propagation, vol. 58, no. 9, pp. 2934-2945, 2010.

[15] T. Takashima, T. Nakae, and R. Ishibashi, "Calculation of complex fields in conducting media," IEEE Transactions on Electrical Insulation, vol. 15, no. 1, pp. 1-7, 1980.

[16] D. Poljak and S. Krešić, "A simplified calculation of transient plane waves in a presence of an imperfectly conducting halfspace," in Proceedings of the 27th World Conference on Boundary Elements and Other Mesh Reduction Methods (BEM 27 '05), pp. 541-550, Orlando, Fla, USA, March 2005.

[17] R. Cicchetti, A. Faraone, G. Orlandi, and D. Caratelli, "Realargument incomplete Hankel functions: accurate and computationally efficient integral representations and their asymptotic approximants," IEEE Transactions on Antennas and Propagation, vol. 63, no. 6, pp. 2751-2756, 2015.

[18] D. Poljak, V. Dorić, F. Rachidi et al., "Generalized form of telegrapher's equations for the electromagnetic field coupling to buried wires of finite length," IEEE Transactions on Electromagnetic Compatibility, vol. 51, no. 2, pp. 331-337, 2009.

[19] D. Poljak, S. Šesnić, and R. Goić, "Analytical versus boundary element modelling of horizontal ground electrode," Engineering Analysis with Boundary Elements, vol. 34, no. 4, pp. 307-314, 2010.

[20] E. D. Sunde, Earth Conduction Effects in Transmission Systems, Dover Publications, New York, NY, USA, 1968.

[21] C. R. Paul, Analysis of Multiconductor Transmission Lines, John Wiley \& Sons, New York, NY, USA, 2008.

[22] L. Grcev and F. Dawalibi, "An electromagnetic model for transients in grounding systems," IEEE Transactions on Power Delivery, vol. 5, no. 4, pp. 1773-1781, 1990. 


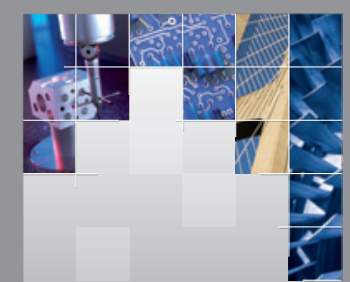

\section{Enfincering}
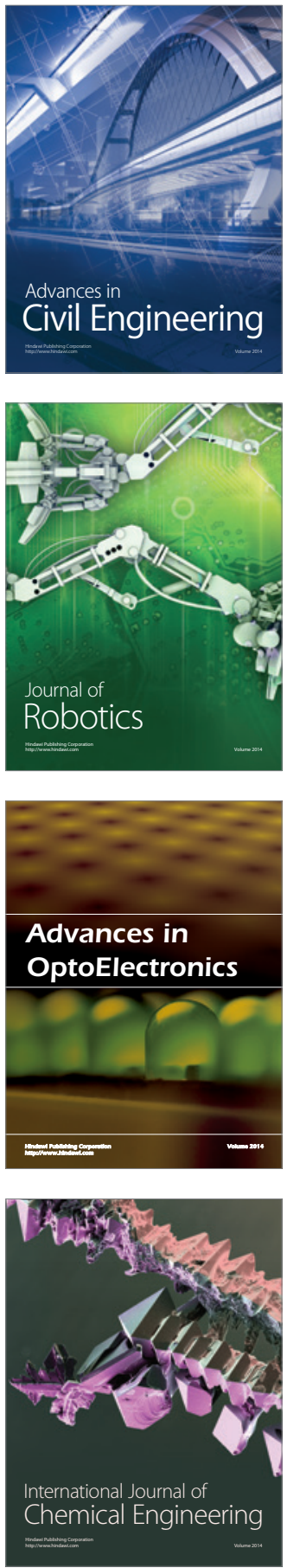

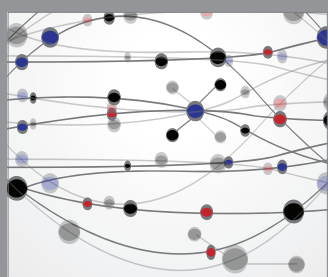

The Scientific World Journal

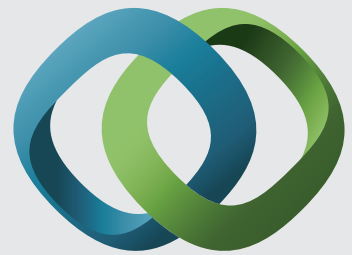

\section{Hindawi}

Submit your manuscripts at

http://www.hindawi.com
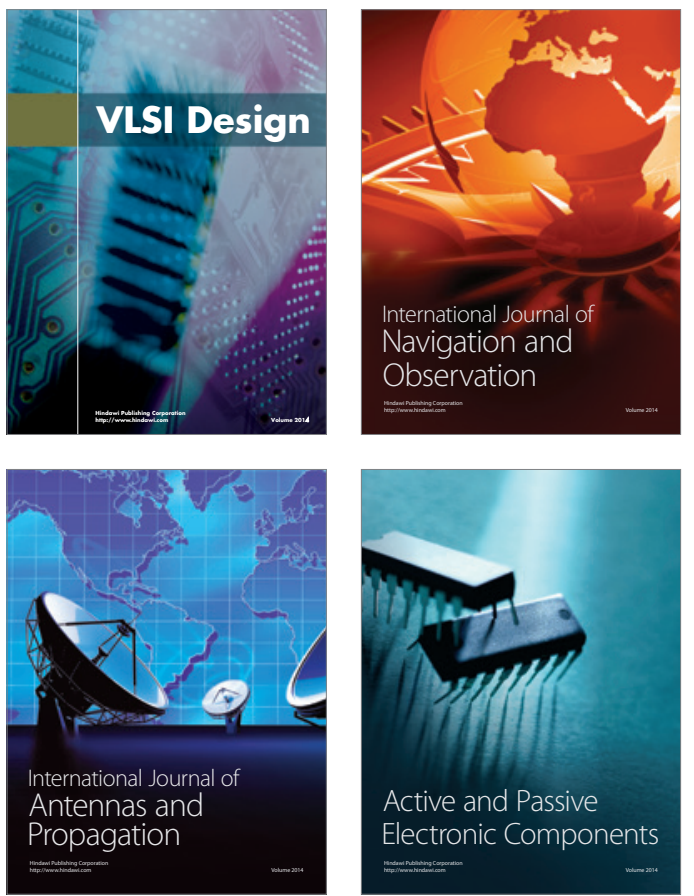
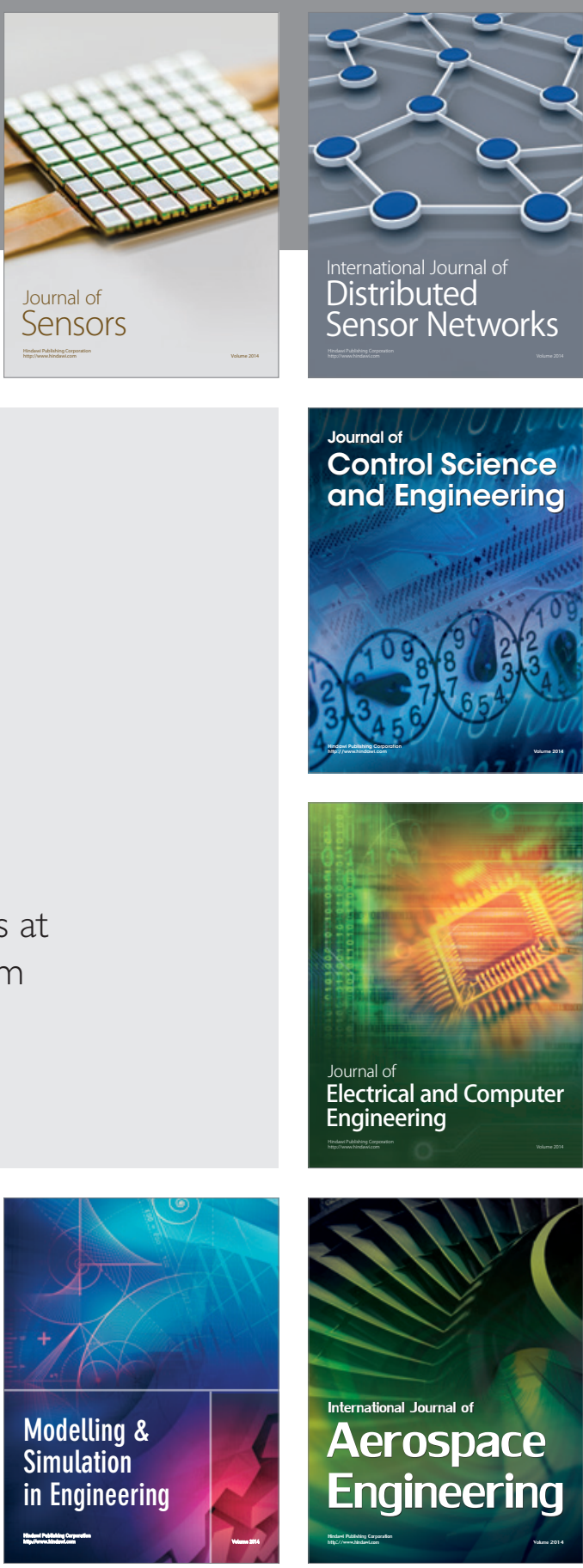

International Journal of

Distributed

Sensor Networks

Journal of

Control Science

and Engineering
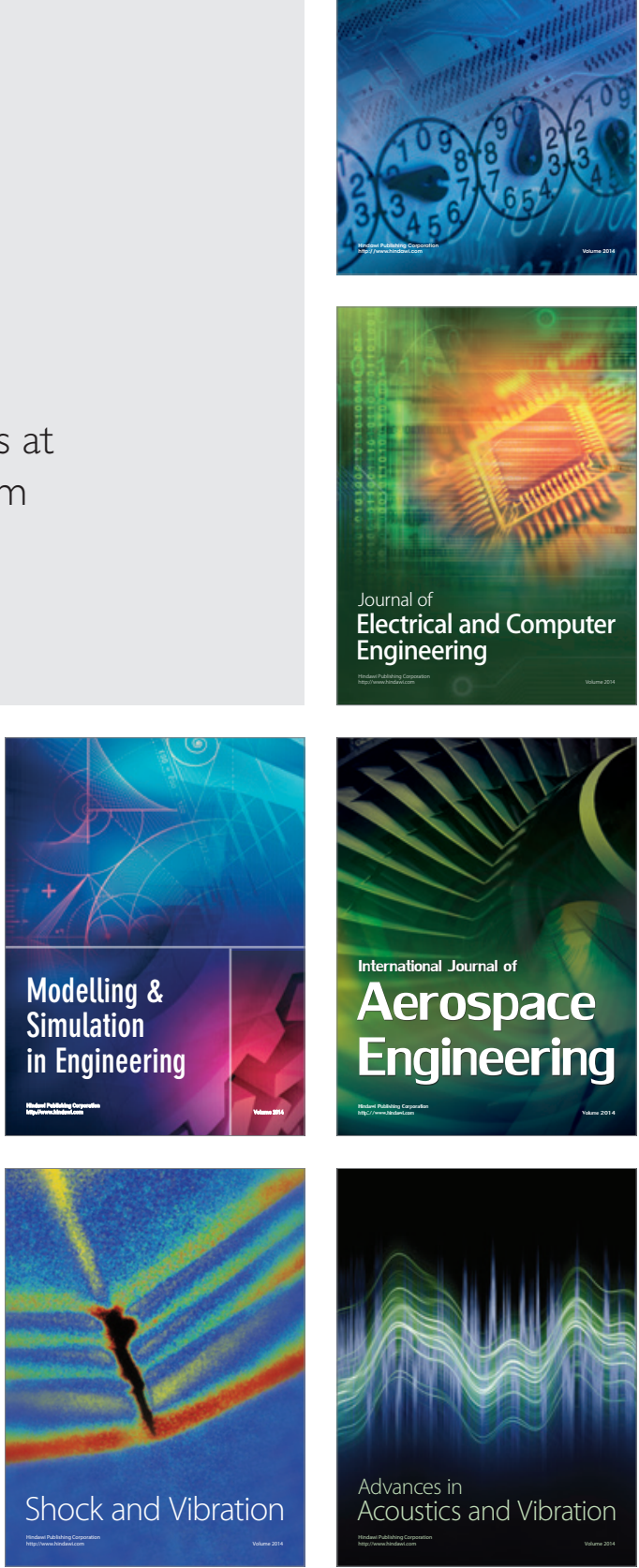